\title{
Co-Occurrence of Guillain-Barre Syndrome and Multiple Sclerosis: A Rare Case Report
}

\author{
Amr Hassan $^{a} \quad$ Alaa El-Mazny ${ }^{a}$ Mohammed Saher ${ }^{b} \quad$ Ismail Ibrahim Ismail ${ }^{c}$ \\ Mohammed Almuqbild, e
}

aNeurology Department, Kasr Al Ainy Hospital, Cairo University, Cairo, Egypt; bNeurology Department, Students Hospital, Cairo University, Cairo, Egypt; ' Neurology Department, Ibn Sina Hospital, Kuwait, Kuwait; dDepartment of Pediatrics, Division of Genetics and Genomics, Boston Children's Hospital, Harvard Medical School, Boston, MA, USA; 'Division of Pediatric Neurology, King Saud bin Abdulaziz University for Health Sciences, Riyadh, Saudi Arabia

\section{Keywords}

Multiple sclerosis · Demyelination · Guillain-Barre syndrome $\cdot$ Medicine $\cdot$ Neurology

\section{Abstract}

Guillain-Barre syndrome (GBS) and multiple sclerosis (MS) are autoimmune demyelinating disorders of the peripheral and central nervous systems, respectively. The co-occurrence of these 2 conditions is rare in the literature. Herein, we present a rare case of GBS and MS in a 19-year-old female who presented initially with GBS followed by MS, and we provide a literature review. Despite being rare, it should be kept in mind in the differential diagnosis of patients with atypical and usual presentation of both diseases.

(c) 2021 The Author(s)

Published by S. Karger AG, Basel

\section{Introduction}

Guillain-Barre syndrome (GBS) is an autoimmune demyelinating disorder of the peripheral nervous system. Multiple sclerosis (MS) is another autoimmune demye- linating disease that affects the central nervous system. Both peripheral and central demyelinating diseases share common immunopathogenic and proposed genetic mechanisms. A nonspecific inflammatory cascade activation occurs, which leads to demyelination, axonal loss, and, consequently, disease progression [1]. The co-occurrence of these 2 conditions is rare in the literature and only reported in very few case reports.

\section{Case Report}

A 19-year-old female, with noncontributory past medical history, experienced watery diarrhea and recurrent vomiting that lasted for 3 days. A week later, she developed acute proximal weakness, tingling, and numbness of the upper and lower limbs with unsteady gait that progressed within 2 days to facial weakness, and she became dependent. Her general examination was unremarkable; her neurological examination revealed bilateral lower motor neuron facial palsy, generalized areflexia, proximal weakness of the 4 extremities of Medical Research Council grade 3/5, negative stretch signs, and loss of deep sensation with sensory ataxia; while

Ismail Ibrahim Ismail has the following ORCiD number: https://orcid.org/0000-0002-9788-7044.] karger@karger.com www.karger.com/dmj

Karger $\stackrel{\text { ' }}{=}$

BOPEN ACCESS
(C) 2021 The Author(s)

Published by S. Karger AG, Basel

This article is licensed under the Creative Commons AttributionNonCommercial-NoDerivatives 4.0 International License (CC BYNC-ND) (http://www.karger.com/Services/OpenAccessLicense) Usage and distribution for commercial purposes as well as any distribution of modified material requires written permission.
Ismail Ibrahim Ismail

Ibn Sina Hospital

PO Box 25427, Gamal Abdel Nasser Street, Sabah Medical Area Safat 13115 (Kuwait)

dr.ismail.ibrahim2012@Gmail.com 


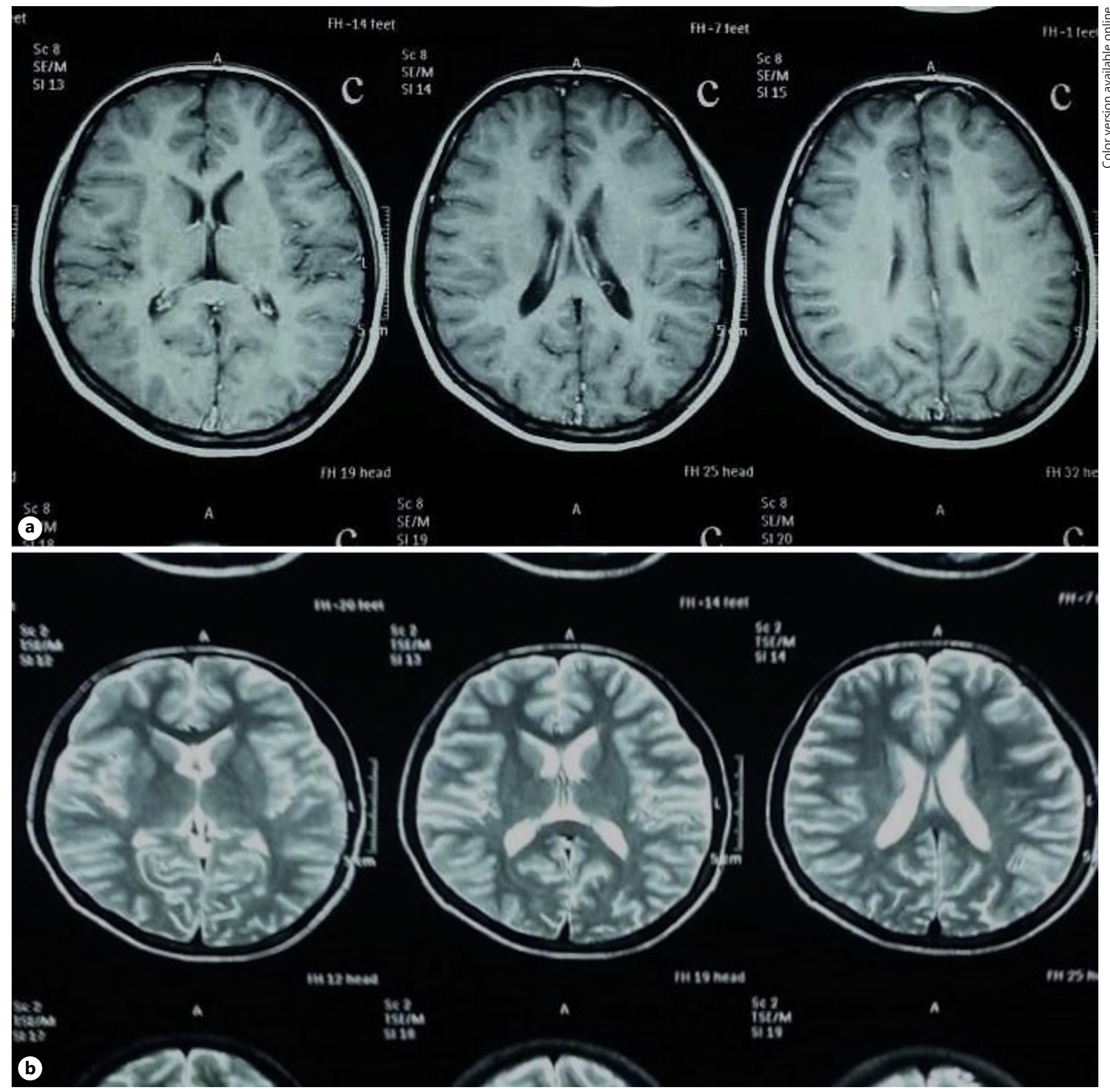

Fig. 1. MRI brain done after the initial presentation revealed no abnormality. a Postcontrast axial T1-weighted MRI. b Axial T2-weighted MRI.

superficial sensory examination was normal. Her initial routine laboratory investigations and nerve conduction study were normal. Lumbar puncture was done on the 3rd day of weakness, and the cerebrospinal fluid (CSF) examination protein was $233 \mathrm{mg} / \mathrm{dL}$, with no lymphocytes and normal glucose. Oligoclonal bands in the CSF and blood were negative. MRI brain and cervical spine with contrast was done, and it was normal (Fig. 1).
Diagnosis of GBS was made on a clinical base, and the patient received 6 sessions of plasma exchange on alternate days, after which the condition regressed to near-complete resolution of the limb and facial weakness. Her follow-up nerve conduction study after treatment showed delay in distal latencies, slowed nerve conduction velocities, and prolonged $\mathrm{F}$ wave supporting peripheral demyelination. Three months later, the patient re-experienced re- 

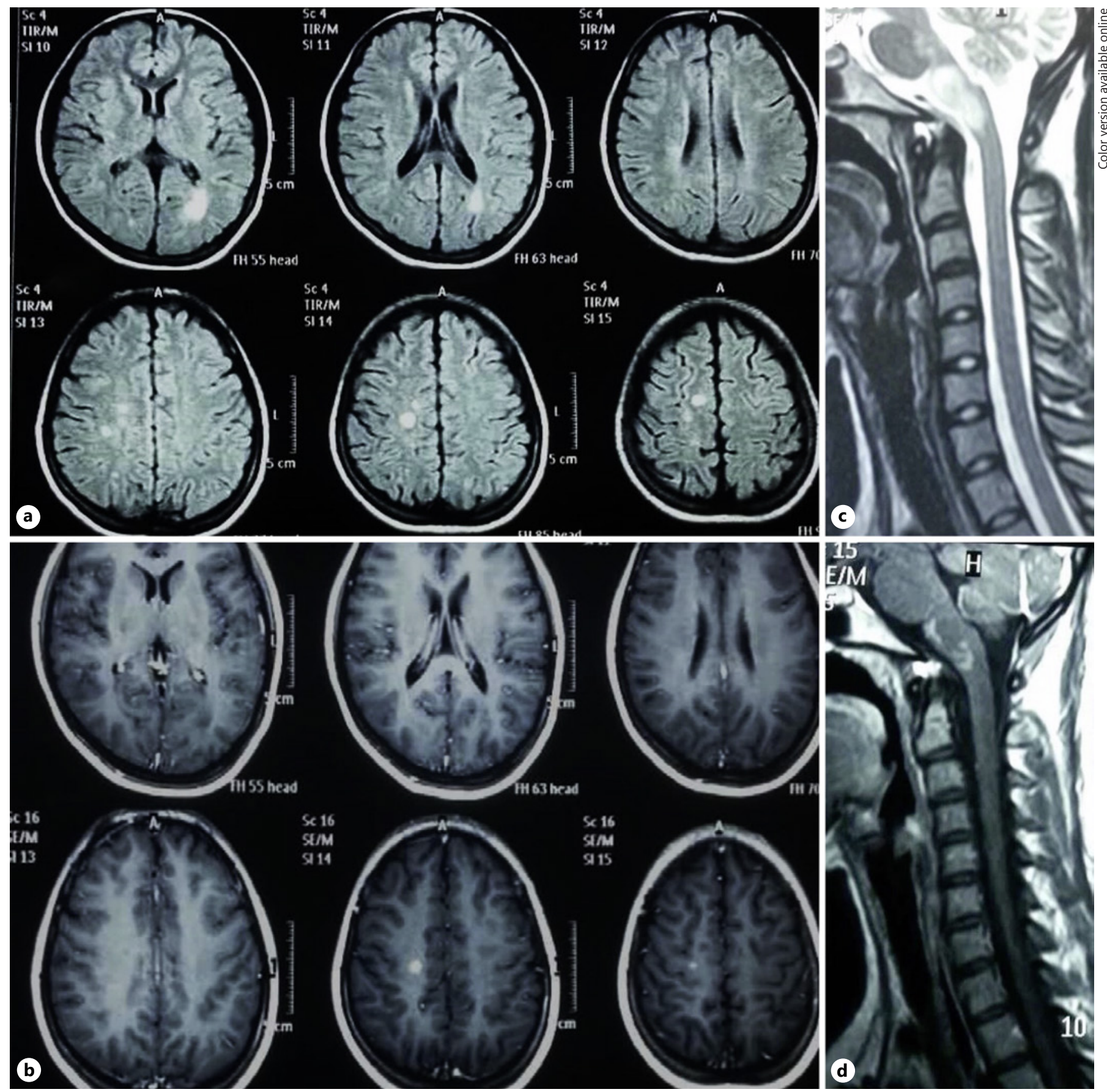

Fig. 2. MRI brain and cervical spine done after the second attack. a Axial FLAIR depicts multiple bilateral periventricular and deep white matter hyperintensities. b Postcontrast axial T1-weighted MRI demonstrates right deep parietal white matter contrast-enhancing lesion. c Sagittal T2-weighted MRI revealed large cervicomedullary hyperintense lesion. d Postcontrast sagittal T1-weighted MRI demonstrating active contrast-enhancing lesion. FLAIR, fluid-attenuated inversion recovery.

peated vomiting for 2 days, with no diarrhea or abdominal pain, and on the 3rd day, she started to re-experience bilateral limb weakness and ataxia that progressed to respiratory distress. The diagnosis of the recurrent form of GBS was considered; therefore, she received 6 sessions of plasma exchange, upon which the patient condition showed marked improvement.

However, a scheduled follow-up MRI brain and cervical spine with contrast was done, and surprisingly, it revealed multiple 
variable-sized bilateral T2-weighted hyperintensities involving the deep white matter and the cervicomedullary junction. Postcontrast studies revealed 2 contrast-enhanced lesions (Fig. 2). Serum anti-aquaporin-4 antibodies (ELISA), ANA, ANCA, antidsDNA antibodies, lupus anticoagulants, B2 microglobulin, anticardiolipin antibodies, C3, C4, angiotensin-converting enzyme, anti-Brucella antibodies, anti-HIV antibodies, and pathergy skin test for Behcet's disease were all negative. CSF analysis revealed elevated IgG index (3.1) and positive oligoclonal bands. One month after her second admission, she developed acute rightsided hemiparesis and hemihypesthesia, with left sixth nerve palsy and bilateral positive Babinski sign. Diagnosis of MS was made, and 1 gram of intravenous methylprednisolone was given for 5 days, upon which the patient noticed marked improvement. She was started on subcutaneous interferon beta- 1 a as a diseasemodifying treatment, and she did not report any further attacks for 18 months.

\section{Discussion}

We described a case of MS that was preceded by GBS. A number of studies in the literature have shown that the co-occurrence of both diseases is more than just a mere coincidence. First, a common genetic link between MS and GBS has been proposed by Vedeler and colleagues [2]. They suggested that certain Fc-gamma receptor $(\mathrm{Fc} \gamma \mathrm{R})$ allotypes, including $\mathrm{FC} \gamma \mathrm{RIIA}$ and FC $\gamma$ RIIIB, were associated with severity of both diseases. However, they could not establish a clear correlation between these allotypes and development of the diseases. Second, Epstein-Barr virus can be considered as a common environmental risk factor for the development of both MS and GBS. Exposure to EBV is well known as a potential risk factor for development of MS and considered as one of the most common infections preceding GBS [3]. Third, from an epidemiological point of view, Langer-Gould et al. [4] in a population-based case-control study showed that MS patients were more likely to develop GBS in their disease course, which was statistically significant when compared with matched controls ( $p=0.006)$. Moreover, GBS preceded MS in 4 of 6 patients in their cohort. Fourth, a common immunopathogenic background was supported by the association of inflammatory demyelinating polyneuropathy (CIDP) and MS and by the occurrence of CNS demyelination following PNS demyelination in EAN animal models [4].

To the best of our knowledge, only 9 patients with concurrent MS and GBS diagnosis have been described in various case report studies, in addition to another 7 patients reported in a population-based survey conduct- ed by Etemadifar et al. [5]. An interesting point in our case is the development of GBS-like presentation in the second attack, which is an unusual presentation of MS, in addition to the co-occurrence with GBS. One of the important differential diagnoses at that stage was recurrent GBS, which can occur in $2-5 \%$ of cases. Another possibility was acute onset of chronic inflammatory demyelinating polyneuropathy. However, the "accidental" discovery of central lesions in the prescheduled MRI, in addition to the third clinical attack, favored the co-occurrence of 2 pathologies in our case. One limitation in this case is not testing for serum antibodies against myelin oligodendrocyte glycoprotein, to fully rule out NMOSD.

In conclusion, despite being rare, the co-occurrence of GBS and MS should be kept in mind by neurologists. The well-established diagnostic criteria must be met to diagnose both disorders. To the best of our knowledge, this is the first case with this unusual presentation of MS and co-occurrence with GBS.

\section{Statement of Ethics}

Written informed consent was taken from the patient.

\section{Conflict of Interest Statement}

There is no conflict of interest by any of the authors in relation to this case report.

\section{Funding Sources}

No funding was received.

\section{Author Contributions}

Dr. A.H.: conception, data acquisition, design, manuscript drafting, critical analysis, and final approval. Dr. A.E.: analysis, review, design, critical analysis, and final approval. Dr. M.S.: analysis, review, design, critical analysis, and final approval. Dr. I.I.I.: corresponding author, conception, data acquisition, review, design, critical analysis, manuscript drafting, and final approval. Dr. M.A.: analysis, review, design, critical analysis, and final approval.
Hassan/El-Mazny/Saher/Ibrahim Ismail/ Almuqbil 


\section{References}

1 Vedeler CA, Matre R, Sadallah S, Schifferli J. Soluble complement receptor type 1 in serum and cerebrospinal fluid of patients with Guillain-Barré syndrome and multiple sclerosis. J Neuroimmunol. 1996;67(1):17-20.

2 Vedeler CA, Myhr KM, Nyland H. Fc receptors for immunoglobulin G: a role in the pathogenesis of Guillain-Barré syndrome and multiple sclerosis. J Neuroimmunol. 2001; 118(2):187-93.
3 Guan Y, Jakimovski D, Ramanathan M, Weinstock-Guttman B, Zivadinov R. The role of Epstein-Barr virus in multiple sclerosis: from molecular pathophysiology to in vivo imaging. Neural Regen Res. 2019;14(3):37386.

4 Langer-Gould A, Albers KB, Van Den Eeden SK, Nelson LM. Autoimmune diseases prior to the diagnosis of multiple sclerosis: a population-based case-control study. Mult Scler. 2010;16(7):855-61.
5 Etemadifar M, Roomizadeh P, Abtahi SH, Sajjadi S, Abedini A, Golabbakhsh A, et al. Linkage of multiple sclerosis and Guillain-Barre syndrome: a population-based survey in Isfahan, Iran. Autoimmune Dis. 2012;2012: 232139 . 